\title{
Trayectorias biográficas de los italianos en el Ecuador de fines del siglo XIX y primera mitad del siglo $\mathrm{XX}^{*}$
}

\author{
Biographical Paths of the Italians in the Ecuador \\ since the End of the 19th Century to the First Half \\ of the 20th Century
}

\section{Chiara Pagnotta}

ORCID iD: http://orcid.org/0000-0002-9810-3109

Universidad de Cádiz

En este artículo pretendo reconstruir las trayectorias biográficas y las redes migratorias del grupo italiano presente en Ecuador a finales del siglo XIX y primera mitad del siglo $X X$. Me interesa aquí poner de relieve los puntos en común entre los diferentes protagonistas de la migración, para esbozar una biografía colectiva —cuantitativamente significativade los inmigrantes italianos en ese país, basada en las fichas consulares recopiladas por las representaciones diplomáticas en Quito y en Guayaquil entre 1897 y 1941.

Palabras Clave: Migraciones; Italianos; Ecuador; Trayectorias.

This article aims to explore the biographical paths and the migratory networks of the Italians settled in Ecuador between the end of 19th century and the first half of the 20th century. To make that, it will focus on the common elements among the various protagonist of the migration. Through the analysis of the registrations of the Italian Consulate in the Diplomatic Representations in Quito and in Guayaquil between 1897 and 1941, It will be realising a collective biography quantitatively significant.

KeYwords: Migrations; Italians; Ecuador; Paths.

Copyright: (C) 2019 CSIC. Este es un artículo de acceso abierto distribuido bajo los términos de la licencia de uso y distribución Creative Commons Reconocimiento 4.0 Internacional (CC BY 4.0).

* Este trabajo se enmarca en el contrato de investigación Marie Courie Fellowship Beatriu de Pinos B (BP-DRG 2013), financiado por la Agència de Gestió d'Ajuts Universitaris i de Recerca (AGAUR) y la Comisión Europea - 7th Framework Programme. 


\section{Introducción}

Los desplazamientos practicados en el tránsito del siglo XIX al XX aparecen caracterizados por la capacidad de los individuos de sobrepasar las fronteras nacionales en número nunca visto antes, gracias a unas cuantas facilidades a la emigración y gracias también a los mecanismos de apoyo y ayuda de las redes de paisanos ya instalados en el exterior.

El caso de la inmigración italiana en Ecuador no es numérica ni cualitativamente comparable con el de otros países americanos, tales como Argentina, Brasil o Uruguay, países cuyas dinámicas internas requirieron en su momento grandes contingentes poblacionales. Ahora bien, un cierto paralelismo se podría establecer entre la inserción/adaptación de los inmigrantes en las sociedades latinoamericanas del lado del océano Pacífico, que han sido afectadas solo marginalmente por los grandes proyectos de migración organizada. Dentro de este panorama, son pocos los estudios que se han ocupado de la zona andina y de los países centroamericanos. ${ }^{1}$

Cabe matizar que en el siglo XIX empezó a delinearse la tensión entre los Estados en formación, para englobar a los actores en el interior de un espacio geográfico definido, sobre el que se ejerciese la soberanía, y sobre los individuos que a menudo tratan de escapar al control de esos Estados protagonizando procesos de movilidad. ${ }^{2}$

Las migraciones del siglo XIX representan un momento de ruptura en la formación/consolidación de aquellos Estados y de sus aparatos, incluyendo el estadístico y el de control sobre la población. Es sabido que en aquel momento, tanto en Europa como en América Latina, la disciplina estadística se desarrolló en diálogo con la construcción del Estado-Nación. ${ }^{3}$ Conforme a las ideas de la época, la ciencia estadística tenía que dar a conocer una realidad — pretendidamente objetiva — sobre la que posteriormente los poderes estatales pensaban intervenir. En síntesis, se trataba de conocer para mejor administrar los nuevos Estados. De otro lado, Benedict Anderson remarca que la ciencia estadística y la geografía fueron también instrumentos para actuar y, en buena medida, moldear los países en construcción. ${ }^{4}$ En esta misma línea se sitúa el trabajo de la historiadora Mercedes

1 Véase Bonfiglio, 1999; Cagiao Vila, 1992; Estrada Turra, 1994; González Martínez, 2003; Devoto, 2007; Martínez Riaza, 2006; Martínez Rodríguez, 2013; Vangelista, 1982.

2 Véase Mezzadra, 2001.

3 A este propósito véase el trabajo de Bustamante, Giraudo y Mayer, 2014.

4 Anderson, 1991. 
Prieto, que expone cómo el censo de población del Ecuador de mediados del siglo XX devino instrumento para la formación de la nación imaginada, creando identidades, inscribiéndolas en categorías, ordenándolas y volviéndolas legibles. ${ }^{5}$

Es oportuno remarcar que Ecuador destaca por caracterizarse por un atraso estadístico desde la independencia y hasta mediados del siglo XX. Si, de un lado, García Moreno promovió la creación de la Oficina Estadística Nacional y la puesta en marcha de un censo cuadrienal, y pensaba traer al país andino a expertos franceses para que enseñaran los métodos y formaran especialistas locales, sus políticas en este sentido alcanzaron escaso resultado a causa de la guerra franco-alemana, que impidió la llegada de los formadores. ${ }^{6}$ En consecuencia, el primer censo nacional en Ecuador se realizó en 1950; con anterioridad hubo únicamente un censo piloto para la ciudad de Guayaquil, en 1897, y otro para la de Quito, en 1907.7

Se entiende que para los estudios de tipo estadístico y demográfico -incluyendo los de extranjería - sobre el Ecuador del periodo, se tenga que recurrir a otras tipologías de fuentes primarias, como los archivos parroquiales y los archivos de policía, entre otros. De manera diferente, en el caso italiano, el interés en cuantificar a los ciudadanos fructificó tempranamente, y el Ufficio Centrale di Statistica fue creado en 1861 (el Regno d'Italia se fundó el mismo año).

Desde un punto de vista metodológico, conviene recalcar que los estudios de Maitron sobre el movimiento obrero francés (y sus militantes, a menudo desconocidos $)^{8}$ han contribuido a superar uno de los límites del método prosopográfico, o de los estudios sobre trayectorias individuales y colectivas, es decir, tener como objeto de estudio privilegiado a las élites o a las capas sociales altas — siendo ellas las que más huellas escritas dejaban, que podían ser estudiadas-, y ampliando el análisis a los grupos sociales subalternos. ${ }^{9}$ Precisamente en esta línea se pretende situar este trabajo sobre un grupo de inmigrantes.

\footnotetext{
5 Prieto, 2015, 30.

6 León, 1871, 9. García Moreno fue presidente de Ecuador entre 1859-1865 y entre 1869-1875.

7 Sobre este tema véase, Pagnotta, 2016, 92-94. El censo de 1950 situaba en 884 el número de los inmigrantes italianos presentes. Censo de población y vivienda, Quito, Instituto Nacional de Estadística y Censo, 1950.

8 Maitron y Pennetier, 1964-1997.

9 Entre otros trabajos sobre el ámbito latinoamericano, véase Schröter y Büschges, 1999.
} 
El objetivo de este artículo reside en intentar entender quiénes eran los inmigrantes italianos en Ecuador, mediante el estudio de los puntos en común entre los miembros de este grupo, cuyas huellas se encuentran en los registros de inscripción de las representaciones diplomáticas italianas en Quito y en Guayaquil. No se trata aquí, por tanto, de ofrecer un conjunto de biografías individuales yuxtapuestas, sino de intentar dibujar algunos rasgos de una biografía colectiva del grupo italiano en Ecuador, basándonos en la observación de las trayectorias de vida de los actores. Se pretende tratar de descifrar la estructura social del colectivo a partir de un análisis de las fichas individuales, para entender quiénes eran los individuos, de qué sector social provenían, las redes de pertenencia y de movilidad, y el entramado en el que actuaban. Sin lugar a duda, se trata aquí de estudiar en clave prosopográfica los vínculos y las relaciones entre los actores sociales protagonistas de la migración. ${ }^{10}$

Por todo ello, las fuentes principales utilizadas para este estudio provienen del Archivo Storico Diplomatico del Ministero degli Affari Esteri, ubicado en Italia (Roma), donde se conservan los registros de los italianos, con las fichas de inscripción, provenientes del Consulado italiano en Guayaquil desde 1897, y de la Embajada italiana de Quito desde 1907. ${ }^{11}$ Los registros abarcan hasta 1941. Con el cierre de las representaciones diplomáticas italianas, debido a la Segunda Guerra Mundial y a la adopción por parte de Ecuador de la doctrina de defensa continental, se suspendió la inscripción de los emigrantes y los intereses italianos en el país andino pasaron a ser representados por la legación de España. ${ }^{12}$

Los datos en nuestras manos corresponden a un total de 875 fichas de inscripción. ${ }^{13}$ Es necesario aclarar que estas fichas son una fuente parcial, ya que no es posible cuantificar exactamente el número de italianos

10 Sobre el método prosopográfico para el estudio de la historia de América, véase Guerra, 1988. Más en general, Stone, 1971.

11 Registro dei Nazionali, Regio Consolato d'Italia a Guayaquil (1897-1937) y Registro dei Nazionali, Ambasciata d'Italia a Quito (1907-1941 y 1946-1971), Archivio Storico-Diplomatico del Ministerio degli Affari Esteri (en adelante ASDMAE). Agradezco a Luigi Guarnieri Calò Carducci que me ha ayudado a ubicar en el archivo estas fuentes — sin número de identificación —; cabe destacar que estas fuentes han sido estudiadas anteriormente por el mismo Guarnieri, 2001, 34-39.

12 La inscripción de los inmigrantes recomenzó en 1946, una vez reanudadas las relaciones diplomáticas. En este trabajo dejamos al margen aquella documentación.

13 Remarco que se trata de una migración cuantitativamente poco numerosa, si la comparamos con el flujo dirigido desde la península italiana hacia el Cono Sur, pero importante si nos ponemos en la perspectiva del país receptor, siendo la italiana, siempre en 1950, una de las nacionalidades extranjeras más numerosas presentes en el país andino, de 23.489 extranjeros en total. 
presentes en el país andino, pues no todos los residentes se inscribían, y sin duda había individuos que evitaban todo tipo de reconocimiento y control gubernamental. ${ }^{14}$ Así, se puede fácilmente entender que no necesariamente todos los inmigrantes italianos presentes en el país andino dejasen sus huellas en dichos registros.

A pesar de que los datos que podemos extraer del estudio de estas fichas pueden ser incipientes, se ha podido avanzar hasta llegar a formar una idea de una tendencia general del fenómeno.

En el primer apartado abordaré los elementos más significativos de las fichas; en un segundo apartado analizaré en detalle las trayectorias que unen la región de Liguria y la costa ecuatoriana; a continuación estudiaré las diferencias regionales y profesionales en las migraciones que unen el sur de Italia y la ciudad de Guayaquil; por último, en el cuarto apartado, indagaré — siempre en su dimensión regional y profesional- los lazos establecidos por los migrantes entre las regiones de Campania y Piamonte y la ciudad de Quito y, más en general, la sierra ecuatoriana. Y concluiré con unas consideraciones de carácter general.

\section{Rasgos generales a través de los registros}

Conforme a lo ocurrido en otros países de América Latina, en el siglo XIX y hasta mediado el siglo XX se verificaron varias tentativas para orientar hacia el país andino los flujos de inmigración europea que se estaban dirigiendo hacia el continente. La promoción gubernamental de la inmigración europea se asociaba a la colonización agrícola acaecida gracias a las compañías (privadas) de colonización.

Dicho esto, nos consta que las políticas pro-inmigratorias de Ecuador se caracterizaron por ser una historia de voluntades, de tentativas incipientes $\mathrm{y}$, finalmente, de fracasos. Aunque se pretendía atraer mano de obra europea para «mejorar la raza» y para hacer progresar al país, teniendo en cuenta que se consideraba que los inmigrantes podrían enseñar los

14 Varios estudios relacionan la emigración con la elusión del servicio militar obligatorio; de esta manera se evitaría cumplir la ley y aparecer en las estadísticas. Para el caso de los ligures, la elusión del reclutamiento se relaciona con el hecho de que el nuevo Estado sabaudo era sentido como ajeno, y a veces enemigo. Cabe remarcar que la anexión de la República de Génova al Reino de Cerdeña se decidió en el Congreso de Viena. Ya en 1868, el periódico genovés La Borsa cifraba en 20.000 los ligures que salieron hacia el Río de la Plata para evitar la conscripción. Cit. en Sanfilippo, $2002,112$. 
métodos de la agricultura europea, los únicos dos proyectos de colonización con población europea que se llevaron a cabo fueron el de la colonia Ayora, en Mindo, poblada con inmigrantes austriacos, y el de la colonia Simón Bolívar, en Saloya, con españoles. Ambas experiencias tuvieron breve vida. ${ }^{15}$

Por otro lado, es cierto que Ecuador no ofrecía grandes atractivos para los inmigrantes que trataran de insertarse en el sector agrícola. Cabe recordar que el sistema de concertaje fue abolido en 1918, sin embargo, después de esa fecha algunos propietarios seguían empleando mano de obra «concertada» y consideraban a los europeos trabajadores inadecuados -léase exigentes-, pues se les suponía incapaces de adaptarse a las condiciones laborales del país andino. ${ }^{16}$ Por parte italiana, en 1862 el cónsul en Guayaquil explicaba que en las regiones interiores de Ecuador residían pocos italianos a causa de la dificultad de encontrar un trabajo convenientemente remunerado. ${ }^{17}$ En 1903 el Commissariato Generale dell'Emigrazione desaconsejaba rotundamente emigrar a Ecuador, ya que no quedaban claras las medidas puestas en marcha para asegurar la ocupación y apropiación de la tierra de los eventuales inmigrantes..$^{18} \mathrm{~A}$ pesar de eso, nos consta por las fuentes oficiales que en 1906 el total de italianos radicados en Ecuador ascendía a alrededor de $700 .{ }^{19}$

Ya en 1891 el cónsul italiano en Guayaquil hacía un llamamiento, desde las páginas del semanario en lengua italiana La Patria. Organo della colonia italiana en el Equatore, para que los italianos residentes en el país andino se registraran en dicho consulado. ${ }^{20}$ Ahora bien, la fecha de abertura del registro en el consulado de Guayaquil se remonta a 1897, mientras que

15 Para un análisis en profundidad de las políticas migratorias ecuatorianas y las tentativas de colonización agrícola, véase Pagnotta, 2016. Sobre la colonia Simón Bolívar, véase Mazeres, 2012; Pagnotta, en prensa [2019].

16 Ponce, 1921, CLXXXVI-CLXXXVII.

17 Francesco Bologna, dal Consulado de Italia en Guayaquil, al ministro de Negocios Extranjeros del rey de Italia, Guayaquil, 17 de abril 1862, Ministero degli Affari Esteri del Regno d'Italia (1861-1887), ASDMAE. Registri copialettere dei dispacci inviati dal Ministero degli Esteri ai vari Consolati nazionali all'estero (491-629), Busta 880, Pacco 242, Rapporti del Consolato di Guayaquil (1861-1868), ASDMAE.

18 «Circolare n. 89. 14 ottobre 1903», Bollettino Emigrazione, 13, 1903. El Commissariato había sido creado por las autoridades italianas en 1901 para velar por las condiciones de los inmigrantes italianos e impedir abusos ya desde el viaje transoceánico y hasta el establecimiento en los nuevos países.

19 Carletti, 1907, 47. Esta y otras fuentes oficiales para los estudios sobre migraciones rara vez incluyen a los inmigrantes sin papeles.

20 La Patria. Organo della colonia italiana en el Equatore, 1891, 1. 
la primera inscripción data de 1899, ocho años después de la primera convocatoria documentada. ${ }^{21}$

En ambos Registros dei Nazionali aparecen los apartados siguientes: 1) fecha [de registro]; 2) nombre, apellido, paternidad; 3) lugar de nacimiento; 4) fecha de nacimiento; 5) profesión; 6) lugar de domicilio en el Reino [de Italia];22 7) lugar de residencia en la jurisdicción del consulado;23 8) justificativo de la inscripción; 9) suscripción del inscrito y del cónsul; ${ }^{24}$ 10) anotaciones eventuales; ${ }^{25} 12$ ) observaciones. ${ }^{26}$

Desde mediados de los años treinta, las informaciones reportadas en los registros se hacen más escasas, y a menudo se anotan únicamente los nombres, apellidos, paternidad, lugar de nacimiento, fecha de nacimiento, profesión y lugar de residencia en la jurisdicción del consulado. Por lo que concierne al registro del consulado de Guayaquil, en unos cuantos casos de fichajes hechos en aquel periodo aparece entre las observaciones una escrita en negro y subrayada en rojo: «raza judía».

Una primera aproximación que es posible hacer a partir de las inscripciones concierne al origen geográfico de la inmigración, ya sea de regiones y territorios italianos o de terceros países, y por año de inscripción en el registro. Emerge un mapa de las relaciones (a veces de matriz colonial) y redes que los ciudadanos italianos —emigrados a Ecuador- y la Italia de aquel entonces tenían establecidas con los demás países. En esta línea, aparece que los vínculos no fueron estrictamente bilaterales, sino incluso trasnacionales. Cabe aclarar que mientras el imperio colonial italiano se fundó después de la invasión de Etiopía (1936), ya desde la época liberal Italia estaba practicando una política expansionista en la orilla oriental del mar Adriático y en África: en 1911 había conquistado Tripolitana y Cirenaica (desde 1934 unificadas bajo el nombre de Libia) y, en 1912, el Dodecaneso; todo ello se refleja en la documentación estudiada (cuadro 1).

\footnotetext{
21 Registro dei Nazionali, Regio Consolato d'Italia a Guayaquil (1897-1937), ASDMAE.

22 En aquel entonces, Italia era una monarquía; la forma republicana fue aprobada por referéndum en 1946.

23 En unos cuantos casos, aquí se encuentra indicado el nombre del connacional o de la empresa italiana que hace de referencia al recién inscrito en Ecuador.

24 En este apartado se encuentran las firmas de los inscritos; las de los cónsules no fueron reportadas en algún caso.

25 Aquí se encuentran las raras indicaciones sobre el parentesco con otros inscritos en el registro.

26 Aquí se encuentran informaciones sobre el año de la partida de Italia.
} 
CHIARA PAGNOTTA

\section{Cuadro 1}

PROCEDENCIA Y PERIODO DE INSCRIPCIÓN CONSULAR ${ }^{27}$

\begin{tabular}{|c|c|c|c|c|c|c|c|c|}
\hline $\begin{array}{c}\text { Región, territorio } \\
\text { o país }\end{array}$ & $\begin{array}{c}\text { N. }{ }^{o} \text { total } \\
(1899- \\
1941)\end{array}$ & 1899 & $\begin{array}{c}1900- \\
1909\end{array}$ & $\begin{array}{c}1910- \\
1919\end{array}$ & $\begin{array}{l}1920- \\
1929\end{array}$ & $\begin{array}{r}1930- \\
1939\end{array}$ & $\begin{array}{c}1940- \\
1941\end{array}$ & $\begin{array}{c}\text { Sin } \\
\text { datos }\end{array}$ \\
\hline Liguria & 210 & 36 & 35 & 79 & 30 & 5 & 2 & 23 \\
\hline Piamonte & 85 & 2 & 8 & 4 & 14 & 21 & 8 & 28 \\
\hline Basilicata & 20 & 2 & 3 & 10 & 3 & 0 & 1 & 1 \\
\hline Marche & 11 & 1 & 0 & 0 & 1 & 0 & 0 & 9 \\
\hline Campania & 68 & 5 & 11 & 17 & 9 & 15 & 0 & 11 \\
\hline Lombardía & 45 & 4 & 6 & 5 & 7 & 7 & 1 & 15 \\
\hline Calabria & 50 & 6 & 11 & 17 & 4 & 5 & 0 & 7 \\
\hline Véneto & 43 & 3 & 6 & 0 & 5 & 11 & 3 & 15 \\
\hline Friul-Venecia Julia & 21 & 3 & 1 & 1 & 2 & 1 & 0 & 13 \\
\hline Cardeña & 9 & 1 & 3 & 2 & 1 & 0 & 0 & 2 \\
\hline Emilia-Romaña & 36 & 0 & 4 & 9 & 7 & 3 & 1 & 12 \\
\hline Sicilia & 17 & 0 & 1 & 0 & 10 & 0 & 0 & 6 \\
\hline Molise & 1 & 0 & 0 & 1 & 0 & 0 & 0 & 0 \\
\hline Lacio & 19 & 0 & 3 & 1 & 1 & 3 & 4 & 7 \\
\hline Toscana & 29 & 2 & 0 & 1 & 9 & 6 & 0 & 11 \\
\hline Pulía & 2 & 0 & 0 & 0 & 1 & 0 & 0 & 1 \\
\hline Umbría & 10 & 1 & 0 & 0 & 1 & 4 & 0 & 4 \\
\hline Trentino-Alto Adigio & 3 & 0 & 0 & 0 & 5 & 0 & 0 & 2 \\
\hline Ecuador & 93 & 3 & 20 & 29 & 18 & 11 & 1 & 11 \\
\hline Perú & 31 & 1 & 8 & 14 & 0 & 1 & 0 & 7 \\
\hline Chile & 6 & 0 & 2 & 1 & 1 & 2 & 0 & 0 \\
\hline Rumanía & 2 & 0 & 0 & 0 & 0 & 0 & 2 & 0 \\
\hline Etiopía & 1 & 0 & 0 & 1 & 0 & 0 & 0 & 0 \\
\hline Argentina & 4 & 0 & 0 & 3 & 0 & 0 & 0 & 1 \\
\hline Libia & 3 & 0 & 0 & 2 & 0 & 0 & 0 & 1 \\
\hline
\end{tabular}

27 Fuentes del cuadro: Registro dei Nazionali, Regio Consolato d'Italia a Guayaquil (18971937) y Registro dei Nazionali, Ambasciata d'Italia a Quito (1907-1941 y 1946-1971), ASDMAE. Elaboración de la autora. 


\section{CUADRo 1 (continuación)}

\begin{tabular}{|l|c|c|c|c|c|c|c|c|}
\hline \multicolumn{1}{|c|}{$\begin{array}{c}\text { Región, territorio } \\
\text { o país }\end{array}$} & $\begin{array}{c}N^{{ }^{\circ} \text { total }}(1899- \\
1941)\end{array}$ & 1899 & $\begin{array}{c}1900- \\
1909\end{array}$ & $\begin{array}{c}1910- \\
1919\end{array}$ & $\begin{array}{c}1920- \\
1929\end{array}$ & $\begin{array}{c}1930- \\
1939\end{array}$ & $\begin{array}{c}1940- \\
1941\end{array}$ & $\begin{array}{c}\text { Sin } \\
\text { datos }\end{array}$ \\
\hline Croacia $^{28}$ & 1 & 0 & 0 & 1 & 0 & 0 & 0 & 0 \\
\hline Francia $^{29}$ & 4 & 0 & 0 & 0 & 1 & 0 & 2 & 1 \\
\hline España $^{30}$ & 2 & 0 & 0 & 0 & 2 & 0 & 0 & 0 \\
\hline Suiza & 2 & 0 & 2 & 0 & 0 & 0 & 0 & 0 \\
\hline Egipto $^{2}$ & 1 & 0 & 0 & 0 & 0 & 0 & 1 & 0 \\
\hline Polonia & 1 & 0 & 0 & 0 & 0 & 0 & 0 & 1 \\
\hline Eslovenia (Istría) $^{32}$ & 2 & 0 & 2 & 0 & 0 & 0 & 0 & 0 \\
\hline Venezuela & 1 & 0 & 0 & 1 & 0 & 0 & 0 & 0 \\
\hline Abruzo & 1 & 0 & 0 & 0 & 1 & 0 & 0 & 0 \\
\hline Colombia & 1 & 0 & 0 & 1 & 0 & 0 & 0 & 0 \\
\hline Panamá & 1 & 0 & 1 & 0 & 0 & 0 & 0 & 0 \\
\hline Sin datos & 31 & 0 & 0 & 2 & 0 & 1 & 0 & 28 \\
\hline
\end{tabular}

Sin lugar a duda, se puede observar una migración diferenciada por regiones de origen, destacando las regiones de Liguria, Piamonte, Campania y Calabria. Dicho esto, el elemento común parece ser la antigüedad de su asentamiento en el país andino. Emblemático en tal sentido es el elevado número de los individuos que componen la segunda generación, es decir, que indican su lugar de nacimiento en Ecuador, aunque de padre italiano.

28 La ciudad de Pola, de la que proviene el único ítalo-croata, perteneció a la República de Venecia hasta 1797, y entre 1918 y 1943 perteneció al Reino de Italia.

29 Cabe indicar que dos individuos, que salieron de Francia rumbo a Ecuador, formaban parte del grupo de italianos de origen judío que se dirigieron hacia Ecuador a consecuencia del régimen fascista italiano y las leyes raciales. A este propósito, véase Capua, 1998. El hecho de que en el registro de Guayaquil, esos dos y otros varios no fueran señalados con la tinta roja, como por el contrario se solía hacer en el registro de Quito, deja abiertos interrogantes acerca de la diferente aceptación de las políticas fascistas por parte del cuerpo diplomático italiano en Quito y en Guayaquil.

30 Ambos con el mismo apellido, indicando el mismo lugar de partida (Madrid) y lugar de residencia en el Reino (Torino).

31 Se puede deducir que la fecha de la inscripción sea posterior a la entrada en vigor de las leyes raciales (la primera es del 5 de septiembre de 1938), ya que se hace su escritura en rojo: «raza judía».

32 Hasta 1797, Istría formaba parte de la República de Venecia. A finales del siglo XIX el territorio era habitado por individuos de lengua italiana, eslovena y croata. 
Cabe matizar que la revolución industrial en Italia se concentró en los territorios del noroeste, en particular en las regiones de Lombardía y Piamonte. Además, los nuevos impuestos fiscales, aplicados por los Saboya después de la unificación, favorecían precisamente la industrialización y desalentaban la agricultura. Todo ello explica que los territorios de partida se encuentren cerca de los centros industriales en expansión, es decir, aquellos lugares que tuvieron un rápido cambio de modelo productivo, lo que motivó que varios sectores «atrasados» —en términos de tipología de producción — practicaran la vía de la emigración.

Antes de entrar más en detalle, tratando de iluminar los vínculos que unen diferentes lugares de origen y de destino, es preciso introducir una aclaración concerniente al género de los inmigrantes italianos presentes en Ecuador.

\section{Cuadro 2}

SEXO Y PERIODO DE INSCRIPCIÓN EN EL CONSULADO ${ }^{33}$

\begin{tabular}{|l|c|c|}
\hline & $1899-1920$ & $1921-1941$ \\
\hline Total & 628 & 241 \\
\hline Hombres & 587 & 192 \\
\hline Mujeres & 41 & 49 \\
\hline
\end{tabular}

Emerge del cuadro 2 que la inscripción de mujeres en el consulado aumenta a partir de los años veinte, mientras que en términos porcentuales disminuye la de varones. Los estudios sobre flujos migratorios italianos hacia América nos dicen que, en términos generales y por lo menos al comienzo, se caracterizan por ser mayoritariamente masculinos. En este sentido, el caso en observación no parece diferenciarse de otros. Ahora bien, sospechamos que esta aparente ausencia femenina puede deberse a una falta de rastros, por parte de las mujeres migrantes, en los archivos gubernamentales, y no a una ausencia femenina tout court en las corrientes de migración. Cabe señalar las razones endógenas a la Italia de la época:

33 Fuentes del cuadro: Registro dei Nazionali, Regio Consolato d'Italia a Guayaquil (18971937) y Registro dei Nazionali, Ambasciata d'Italia a Quito (1907-1941 y 1946-1971), ASDMAE. Elaboración de la autora. 
en el Código Civil de 1865 se sancionaba la inferioridad femenina respecto al hombre, y únicamente en 1975 fue abrogada la potestad marital. Si el sufragio femenino fue introducido en Italia en 1946, en Ecuador era vigente desde 1929. Por todo ello es fácil intuir que — por la época- la inscripción en la sede de las representaciones diplomáticas la hacía quien ejercía la patria potestad sobre todo el núcleo familiar.

Sin embargo, el hecho de que las primeras mujeres inscritas fueron, entre 1907 y 1908, las hijas nacidas en Ecuador ${ }^{34}$ de aquellos italianos de origen ligur — que se habían asentado en Guayaquil y se habían conectado rápidamente con el mundo empresarial, liberal y, a menudo, masónico del puerto_- ${ }^{35}$ nos habla del recorrido cultural e ideológico de este grupo.

Es adecuado matizar que, hasta la Primera Guerra Mundial, Ecuador fue protagonista de una gran bonanza económica, marcada por la exportación cacaotera. El auge del cacao conllevó la expansión de la frontera agrícola en la costa y el aumento del poder económico y político de los terratenientes que allá tenían sus propiedades. A raíz de las exportaciones, se fue desarrollando el sector comercial y banquero. Guayaquil se convirtió en el centro económico del país y puerto principal para las exportaciones de productos destinados a la comercialización en el mercado mundial. ${ }^{36}$ Por todo ello se explica que el puerto fuera elegido por los inmigrantes italianos como principal lugar de asentamiento y/o actividad económica.

Cabe subrayar que en los registros aparecen 508 inmigrantes que han indicado Guayaquil como lugar de domicilio en Ecuador y, de ellos, 251 declaran tener una actividad relacionada con el comercio. Entre las categorías profesionales reportadas se señalan: 222 comerciantes, 8 empleados de comercio, 5 industriales, 3 comerciantes ambulantes, 2 comerciantes y poseyentes, 2 comerciantes y terratenientes, 2 negociantes, 2 constructores navales, 1 albergador, 1 procurador de una firma, 1 viajante de comercio, 1 representante de comercio, 1 comerciante, fabricante y terrateniente. ${ }^{37}$

34 La ficha de inscripción reporta la firma del padre para las hijas recién nacidas o/y menores de edad.

35 Sobre algunas figuras de la élite italiana en Ecuador, véase Estrada, 1993.

36 Quintero y Silva, 2001, 193-217. Ayala Mora $(2011,37)$ afirma que los agroexportadores no representaban una burguesía moderna, más bien se trataba de unos cuantos terratenientes tradicionales en cuyo seno habían surgido comerciantes y banqueros. Según Quintero y Silva (2001) la que definen como burguesía comercial bancaria estaba formada por la clase terrateniente cacaotera y los comerciantes del puerto de Guayaquil.

37 Elaboraciones de la autora a partir de los datos encontrados en Registro dei Nazionali, Regio Consolato d'Italia a Guayaquil (1897-1937) y en Registro dei Nazionali, Ambasciata d'Italia a Quito (1907-1941 y 1946-1971), ASDMAE. 
Ahora bien, la especialización profesional declarada en la inscripción es sumamente ambigua y subjetiva, ya que en la categoría de comerciante, a falta de mayores especificaciones, podrían entrar tanto los pequeños comerciantes al detalle como los grandes exportadores, habiéndose rastreado la presencia de los italianos tanto entre los grandes beneficiarios del cacao como entre los pequeños minoristas. ${ }^{38}$

Como segundo destino de la inmigración italiana en Ecuador aparece la ciudad de Quito, llegando a ser indicada como lugar de residencia por 194 individuos. Se trata de un destino mucho menos atractivo que Guayaquil. En todo caso, un par de elementos destacan inmediatamente. La primera fecha de inscripción es de $1903 .{ }^{39}$ A partir del siglo XX son muy escasas las informaciones sobre la fecha de partida de Italia, así que este dato no puede ser considerado fiable. Lo que aparece con claridad es una diferencia en cuanto al lugar de origen, siendo 38 piamonteses, 28 de la Campania y 24 nacidos en Ecuador, y los de la Liguria únicamente 6. La diferenciación geográfica va a la par con la profesional, cubriendo un abanico bastante más amplio que el caso anterior. ${ }^{40}$

Junto con las dos grandes ciudades del país, se destacan Ambato y Cuenca como meta de la migración de religiosos. Se trata, en el primer caso, de los capuchinos, diez entre religiosos y personal de la misión, que se registraron en el consulado en Quito en 1930. En este grupo se anotan dos sacerdotes y dos obreros provenientes de Roma, y seis misioneras de Turín. En el caso de Cuenca se trata mayoritariamente de los salesianos; son 24 religiosos, 8 provenientes de Roma y 16 de Turín, y entre ellos se destaca la presencia de cinco religiosas. En ambos casos se indica el lugar de domicilio, que corresponde a la casa madre del instituto religioso de pertenencia. El origen de los misioneros capuchinos era prevalentemente el Véneto, y el de los salesianos era más variado.

Un ulterior elemento de carácter general destaca del estudio de las fichas. Aunque la mayoría de las inscripciones, en particular de quienes provenían de la Liguria, se hicieron en las primeras décadas de existencia

38 Como la familia Parodi, de la que hablaremos más en detalle. En todo caso, cabe subrayar que en el registro consular de Guayaquil el «fundador de la dinastía» aparece únicamente como «comerciante».

39 Se encuentra en el Registro dei Nazionali, Regio Consolato d'Italia a Guayaquil (18971937), ASDMAE [sin número de página]. Se trata de Francesco Ferro Basile, un comerciante oriundo de Reggio Calabria, nacido en 1839.

40 Véase nota 37. 
del registro en Guayaquil, es cierto que la fecha de la emigración es anterior, revelando un flujo constante en el tiempo a partir de la segunda mitad del siglo XIX. ${ }^{41}$ Sobre un total de 271 indicaciones que contienen el año de partida de Italia, o de un tercer país, rumbo a Ecuador, hay 113 individuos que viajaron antes de $1890 .^{42}$ Los estudios genealógicos de Noboa parecen confirmar la temprana migración italiana hacia la ciudad de Guayaquil, situando en alrededor de cien individuos los italianos presentes en el puerto en $1855 .{ }^{43}$ Todo ello significa, además, que la conexión formal entre inmigrantes italianos y madre patria se daba unos cuantos años después del asentamiento, dejando así entender que la relación con las instituciones del país de origen se caracterizó por tener un perfil bajo, que iba a la par con el hecho de que las instituciones italianas de la época se mostraban poco preocupadas por la suerte de los emigrantes. ${ }^{44}$

\section{El comercio y los ligures}

Parece que fue el contexto ecuatoriano el que moduló la vida en el lugar de llegada. Se puede afirmar que un buen número de italianos presentes en Ecuador lograron insertarse en la economía relacionada con los comercios que tenían su eje en el puerto del país. Según el cónsul Goding, los italianos en el puerto habían alcanzado un nivel tal que representaban, junto con los alemanes, los principales participantes en la distribución al por mayor y por menor. Destacaban en particular familias italianas tales como Parodi, Bruzzone y Cía., Cassinelli Hnos. y Cía., Castagneto y Cía., Frugone y Cía., Antonio Baudino, y Damián Miranda. ${ }^{45}$ La Sociedad Geográfica Italiana testifica que serían los italianos — cuyos componentes predominantes eran los de origen genovés - el grupo extranjero más rico asentado en el puerto. ${ }^{46}$

41 Desgraciadamente, no ha sido posible realizar un estudio exhaustivo sobre este dato, debido a que las informaciones sobre el año de emigración a veces no son reportadas.

42 En unos cuantos casos en que la migración no se ha dirigido inmediatamente de Italia a Ecuador, se reporta la fecha de partida de Italia y la eventual fecha de salida de un país tercero antes de la llegada a Ecuador.

43 Jurado Noboa, 2002.

44 Recordamos aquí cómo, en todo caso, Italia empezó a preocuparse de sus inmigrantes en 1901, cuando se promulgó la primera ley de emigración. Véase Ostuni, 2001.

45 Crawford de Roberts, 1980, 108.

46 Annoni, 1920. 
Es sabido que los genoveses fueron los primeros en partir hacia América Latina debido al hecho de que Génova era el principal puerto de partida hacia aquel territorio. ${ }^{47}$ En la mitad del siglo XIX, la ciudad sirve como puerto para un amplio territorio que comprende Liguria, Emilia, Toscana, Piamonte y Lombardía. ${ }^{48}$

El caso de la emigración de la familia Parodi a Guayaquil, y de la red de circulación que se crea alrededor de esta familia, es particularmente iluminador al respecto, y permite apuntar unas cuantas conclusiones de ámbito más general a partir del estudio de su historia y trayectoria migratoria. ${ }^{49}$

El primer miembro del que podemos rescatar las huellas en los registros deja Italia en 1871 rumbo a Ecuador. Se trata de Agostino. El mismo año llega a Ecuador Giovanni Amedeo, de dos años de edad, junto con su familia. ${ }^{50}$ En 1892 llega Domenico. Los tres son originarios de Sanremo, en el poniente ligur. ${ }^{51}$ Domenico y Agostino se inscriben en 1899, y Giovanni Amedeo en 1910, los tres como comerciantes asentados en el puerto de Guayaquil.

La segunda generación de los Parodi (hijos de Giovanni) nacida en Ecuador (Umberto nace en 1897, y Ernesto en 1899), sigue manteniendo un vínculo con la madre patria, indicando Sanremo como lugar de domicilio en el Reino, aunque Ernesto se registra en 1930 y Umberto en fecha desconocida, entre 1925 y 1926. Ambos hijos parecen poder viajar fácilmente entre Ecuador e Italia, ya que, aunque nacidos en Ecuador, viajaban con el pasaporte emitido por el prefecto de Sanremo, en el primer caso, y por el cónsul honorario de Sanremo, el Dr. Parodi, en el segundo. ${ }^{52}$ De la segunda generación que desarrolla parte de su vida en Ecuador forma parte un tercer hermano, Enrico, nacido en Sanremo en 1904, que viaja a Ecuador en 1928.

47 Sori, 1979, 295.

48 Para una síntesis reciente sobre las características de la emigración italiana, véase Colucci y Sanfilippo, 2010.

49 Otras fuentes indican una presencia más antigua de los italianos de este apellido. El 31 de octubre de 1820 aparecen cinco italianos en un listado de extranjeros europeos residentes en Guayaquil, que donaron 16.000 pesos para las «urgencias de la patria»; se atestigua que Antonio Parodi donó 200 pesos. Estrada, 1993, 19.

50 Desconocemos la relación de sangre entre los dos y si viajaron juntos, ya que en este caso solo se reporta en el registro de Guayaquil el año de salida.

51 Un documento de 1734 estudiado por Giovanni Levi (1973) señala que desde el poniente ligur existía un flujo de emigración de faquines hacia el puerto de Cádiz. Según Molinari (1995), la crisis de las actividades del puerto de Cádiz motiva a los ligures a embarcarse para América del Sur.

52 Estrada $(1993,40)$ dice que Giovanni se casó en 1878 con Luisa Goetta, vecina de Sanremo. Los hijos de la pareja fueron: Luis, Mercedes, Humberto y Ernesto, nacidos en Ecuador, y Julio, Emilio, Enrico y Yolanda, nacidos en Italia después del regreso del jefe de familia a Sanremo en 1899. 
El comercio fue la dedicación inicial de los Parodi. Giovanni se conecta con otros connacionales y, en particular, con Emilio Marengo, otro ligur, nacido en Génova, y juntos abren una tienda de productos de exportación, «El Indio Guayas», en Guayaquil. Esta tienda estaba dedicada a la importación directa de abarrotes, ferretería y combustibles de Europa y Estados Unidos, y a la venta en Guayaquil al por mayor y menor. ${ }^{53}$ A Giovanni Marengo se une en Guayaquil un hermano suyo, que anteriormente había emigrado a Perú.

Se puede afirmar que el despliegue económico de algunos de los pioneros, como Parodi, estuvo vinculado a las actividades de exportación en el puerto de Guayaquil, que les permitió acumular capital y reinvertirlo en otros ámbitos, diversificando sus actividades. Gracias al análisis de las fichas, se entrevé un ascenso social de la primera a la segunda generación. La segunda generación no es adscrita como comerciante, sino como terrateniente y comerciante (Umberto), y acomodado (Ernesto). Sabemos que el pionero Giovanni vuelve a vivir en Sanremo y deja a los hijos tres haciendas agrícolas — que había adquirido en Guayas - para que las administren: Roma, Italia y Concepción. ${ }^{54}$ Estas haciendas conjuntamente sumaban un millón y medio de plantas de cacao y a comienzos del siglo XX se estaban desarrollando en ellas plantíos de café y banano. A las primeras tres haciendas se sumaron otras dos: El Carmelo, en la Isla de Puna, y La Alegría, en Guayas. ${ }^{55}$ Nos consta que la llegada de Enrico a Ecuador en 1928 fue precisamente para hacerse cargo de las haciendas de la familia, por pedido del padre, Giovanni. El mismo Enrico además invierte parte del capital en la puesta en marcha de una fábrica de enlatados de atún y sardinas y una fábrica de botones de tagua. ${ }^{56}$

Aparece aquí una «dinastía» completamente insertada en la producción para la exportación en el mercado mundial, que llega a conectar la pequeña ciudad de Sanremo con el comercio transoceánico. Sabemos que de los dieciocho individuos que indican sus domicilios en el Reino en la ciudad de Sanremo, los primeros en llegar a Ecuador han sido Giovanni Amedeo, en 1870, y Agostino, en 1871. Los otros llegaron en el siglo XX. Consta en el registro de Guayaquil que, de las dos mujeres del grupo, Caterina Emanuela Rambaldi se casó en 1926 con un tal Umberto Parodi (no

53 Panfleto publicitario de la tienda. Estrada, 1993, 66.

54 Aliprandi y Martini, 1935, 46.

55 Estrada, 1993, 40.

56 Dizionario storico biografico dei Liguri, 2006, II, 106. 
tenemos mayores informaciones sobre el), y Zelinda Ida Repetto estaba casada con otro paisano, un tal Giobatta Almerini, que formaba parte de los inscritos; hay un escultor que se dirigió a Riobamba, y todos los demás se instalaron en Guayaquil. Entre los hombres, doce tenían actividades conectadas con el comercio. Como era frecuente, y como es por ejemplo el caso de los Parodi, la administración de las propiedades o la vivienda principal estaban en Guayaquil, aunque las haciendas se situaban en otras partes.

Siguiendo siempre las huellas dejadas por los Parodi, encontramos a Giuseppe, un primo de Giovanni, propietario de la hacienda San Pablo en la parroquia de Naranjal (provincia del Guayas). En las primeras décadas del siglo XX era una de las más florecientes del país, y ocupaba una extensión de alrededor de 4.000 hectáreas entre plantíos de cacao, banano y terrenos para la cría de ganado. Igual que su primo Giovanni, Giuseppe, después de una experiencia ecuatoriana, había vuelto a Sanremo, dejando la hacienda bajo la administración de un pariente, en este caso su yerno, Luigi Repetto. ${ }^{57}$

Cabe subrayar que Giovanni, una vez de regreso a su tierra natal, a caballo entre el siglo XIX y el XX, tuvo el cargo de cónsul honorario de Ecuador en Sanremo. Podemos entender que tal distinción, otorgada por el gobierno ecuatoriano, representaba un cargo más simbólico que real, ostentado para representar el ascenso social logrado en la tierra de emigración. De otro lado, el capital económico y político adquirido en tierra ecuatoriana revirtió políticamente en Italia. Las fuentes diplomáticas atestiguan que un tal Ernesto Parodi, nacido en Ecuador, fue un alto cargo de la sección del fascio de Sanremo, y fue el propulsor de un monumento - que nunca vio la luz - de homenaje y amistad entre Italia y Ecuador, aunque entre los opositores al proyecto se encontraban sus mismos hermanos. ${ }^{58}$ Contemporáneamente, varios miembros de la familia Parodi en Ecuador se habían fusionado con el mundo local, siendo parte ya de los grupos económico y financiero y de la élite étnica. Formaban también parte de la Società italiana di Beneficienza «Garibaldi» (Giuseppe fue fundador y tesorero de la primera junta), de la Cámara de Comercio ecuatoriana y del Rotary Club.

Todo ello pone de relieve cómo esta red familiar y de paisanaje se ha consolidado como grupo de poder; con el capital acumulado en la actividad

57 Aliprandi y Martini, 1935.

58 El tema es objeto de otro artículo de la autora: Pagnotta, en prensa [2020]. 
comercial en el puerto se arriesgaron a entrar en otros ámbitos, raras veces abiertos a los extranjeros. Es evidente, a partir del caso de los Parodi, que unos pocos pioneros se insertaron en el mundo económico ecuatoriano durante una coyuntura favorable debida a la bonanza del comercio del cacao, que duró desde finales del siglo XIX hasta la Primera Guerra Mundial.

Cabe subrayar que a comienzos del siglo (en el quinquenio 19001905) se exportaban a Italia, principalmente, cacao, tagua y café, mientras que en 1906 se importaban por Guayaquil artículos como tejidos, vermut, sombreros, aceite, mármol y otros varios productos alimentarios y maquinarias, estando presentes en la ciudad de Génova sesenta y una casas de comercio italianas que tenían relación con Ecuador, ${ }^{59}$ denotando así un floreciente espacio para el consumo de productos que llegaban de Europa y las posibilidades de inserción en un sector florido.

Los intereses económicos comerciales de los Parodi se conectaban también con el levante ligur y sus redes migratorias. Sabemos que a comienzo del siglo XX Giovanni Rovere —oriundo de Sestri Levante- estaba ejerciendo como administrador de la hacienda Italia de Giovanni Parodi. ${ }^{60}$

Este no es un caso aislado, ya que se encuentran, provenientes de la zona costera de levante, 12 individuos de Camogli (10 comerciantes), 33 de Santa Margherita Ligure (tres comerciantes y terratenientes, dos empleados, 25 comerciantes), 31 de Rapallo (18 comerciantes, 2 empleados, 1 tendero), 8 de Chiavari (4 comerciantes, 1 agricultor, 1 capitán de marina, 1 empleado, 1 terrateniente), 7 de Cogorno, en las alturas de Chiavari (los siete comerciantes). Todos ellos se asientan en Guayaquil. ${ }^{61}$

Como ha subrayado Adele Maiello, la zona del levante ligur y, de manera particular, de Chiavari, fue el primer lugar de partida de la emigración de la región Liguria entre 1876 y 1915, si relacionamos el número de emigrantes con el número de población total. ${ }^{62}$ En todo caso, se trataría aquí de una práctica de movilidad de tradición antigua. Conforme a lo afirmado por Marco Porcella, la emigración estacional y ambulante era un fenómeno endémico del territorio de Chiavari y sus alrededores. Esta era practicada ya desde el Antiguo Régimen y fue activa en abrir las puertas a una posterior, en masa, a larga distancia, hacia América. ${ }^{63}$

59 Mera, 1907. (J.T. Mera era cónsul general del Ecuador en Italia, con residencia en Génova).

60 Aliprandi y Martini, 1935, 48.

61 Véase nota 37.

62 Maiello, 1986.

63 Porcella, 1989. 
Se finaliza este apartado destacando cómo las partidas hacia Ecuador no concernían únicamente a las dos riberas ligures, sino que se partía también de la ciudad de Génova. Del centro de Génova ${ }^{64}$ salieron 26 individuos, entre ellos ocho comerciantes y varias otras diferentes figuras profesionales típicas de una zona urbana: un fabricante de sombrillas, un empleado comercial, un estudiante, un frutero, un empleado, un fabricante de muebles, un industrial, un vendedor, un contable, una menor (una niña que vive con su padre), dos agricultores, un albañil, un escultor, un maestro albañil, un doctor contable. ${ }^{65}$ Veinte de estos viven en Guayaquil; el primero en vivir en Quito, en 1907, es el escultor. Desde Nervi salieron nueve, y entre ellos un capitán naval, dos capitanes marítimos y seis comerciantes, asentándose todos en Guayaquil. ${ }^{66}$

\section{Una fábrica de hombres para uso ajeno ${ }^{67}$}

Aunque de menor importancia numérica, otros casos son particularmente significativos para entender las dinámicas y las trayectorias migratorias de los italianos en Ecuador.

La atención es capturada por la dimensión local del flujo que se conecta con el ámbito profesional y el capital económico. Es sabido que las poblaciones rurales del sur de Italia se sumaron a los grandes flujos migratorios transoceánicos solo posteriormente, a partir de la última década del siglo XIX. El nacimiento de Italia como Estado conllevó una serie de cambios dirigidos a la modernización del país, que se dieron primero en el norte y luego en el sur. Por lo que concierne al meridione de Italia en época post-unitaria, una primera fase de políticas liberales tuvo como consecuencia que las manufacturas de la zona fueran derrotadas por las provenientes del exterior. Una fase posterior, más proteccionista, dañó las exportaciones de los productos agrícolas locales ${ }^{68}$ Todo ello determinó un incremento de las emigraciones del sur, y además un cambio en los sectores sociales de origen, predominando ahora una migración de origen campesino. En poco

64 En 1926 se conformó la Génova actual, anexando 19 municipios externos al núcleo central de la urbe.

65 Posiblemente un licenciado contable.

66 Véase nota 37.

67 Braudel, 2010, vol. 1, 37.

68 Corti y Sanfilippo, 2012, 82-85. 
tiempo, el puerto de Nápoles superará al de Génova en cuanto a número de embarques. ${ }^{69}$

El flujo proveniente de la región de Calabria amplía el abanico interpretativo de la tipología de la inmigración italiana en Ecuador. En el caso en cuestión, se ve claramente el origen local y del piedemonte del flujo, que sobrepasa los confines regionales calabreses para abarcar dos núcleos situados en el ecosistema socio-económico del Parque del Pollino (de la cadena montuosa de los Apeninos meridionales) ${ }^{70}$ del lado de la región de Basilicata: se trata de Castelluccio Inferiore y Castelluccio Superiore. El flujo hacia Ecuador parece surgir de manera preponderante del lado calabrés de la montaña, de los municipios de Santa Domenica Talao, Morano Calabro, Orsomarso y Mormanno, que en términos administrativos forman parte de la provincia de Cosenza. Sobre los 50 casos de emigración desde la Calabria que aparecen en los registros, 37 provienen del territorio conformado por los cuatro municipios. ${ }^{71}$ Por todo ello, parece que el Ecuador haya entrado a ser parte de aquella estrategia de movilidad y de especialización laboral de las poblaciones del Pollino que caracteriza el Antiguo Régimen y la Edad Contemporánea. Aunque la mayoría de los calabreses presentes en Ecuador se dediquen, como en el caso anterior, al comercio y se establezcan en Guayaquil, aparecen unas cuantas indicaciones de que podría tratarse de una migración de menor capital cultural, figurando en algunos casos - entre las anotaciones - que las personas inscritas son iletradas. Emergen, además, unas figuras profesionales características de la emigración de aquel territorio de origen, y que parecen haberse asentado en Ecuador empleándose en el mismo sector profesional. Al lado de los comerciantes, se encuentran artesanos: bisuteros, orfebres, toneleros, zapateros, sastres. Aparecen además tres vendedores ambulantes.

El historiador Cappelli sitúa la ciudad de Maratea dentro de la misma historia y estrategia emigratoria de un flujo de artesanos, aunque la ciudad se encuentre en el litoral lucano. Cappelli sigue las huellas de un flujo constante de artesanos, especializados en trabajar el oro, la plata y el estaño, que desde este pequeño núcleo de Basilicata parten con destino a Francia, España y Bélgica, y a América Latina, orientándose en particular hacia el Distrito Federal y las regiones centrales de México; Bogotá y Puerto Buenaventura,

69 Bianchi Tonizzi, 1992, 26.

70 Sobre la emigración del Pollino véase Cappelli, 2005.

71 Véase nota 37. 
en Colombia; San Fernando de Apure y Ciudad Bolívar, en Venezuela; unos cuantos más a Panamá, para trabajar en la abertura del Canal; y otros a Guayaquil y otros lugares de Ecuador. ${ }^{72}$ En este último caso, que es el que aquí nos ocupa, en los registros aparecen inscritos dos doradores que desde Maratea se dirigieron a Calceta, en Manabí. Además hay constancia de un hombre, Biagio Laprea, comerciante y sastre a medida, que se instala en Quito trabajando para la casa Visconer con su hijo menor de edad. ${ }^{73}$ También provenientes de Maratea son otros dos comerciantes que se asientan en Guayaquil. ${ }^{74}$

En el caso de la región de Basilicata, la fecha de salida más antigua se remonta a 1884 y corresponde a un tal Francesco Paolo Cataldo, un comerciante oriundo de Castelluccio Inferiore y residente en Vinces, que se registró en el consulado en 1899. Cabe recordar que por aquella época Vinces —llamada la «pequeña París»— era el eje del auge del cacao y — gracias al éxito del comercio - los propietarios de las haciendas vivían entre Ecuador y Francia, educando a sus hijos en las escuelas francesas y trasladando costumbres y arquitectura de aquel lugar al territorio de origen. ${ }^{75}$ En los registros aparecen otros oriundos de los mismos territorios de Francesco Paolo Cataldo. Lo que resalta, sin lugar a duda, de la emigración de las dos provincias, Potenza y Matera, que componen la región de Basilicata, es la hegemonía absoluta de la emigración desde la primera, quedando en cambio totalmente ausente la marcha desde la otra. ${ }^{76}$

Parece así que la emigración hacia Ecuador haya sido practicada desde lugares más pequeños y, en general, del interior de la región, con la única exclusión de las seis salidas de Maratea. La única emigración de la ciudad de Potenza, capital de la provincia homónima, es la de un comerciante, un tal Decio Martino, que se estableció en Quito. ${ }^{77}$

\section{Hacia el interior, en el siglo $\mathrm{XX}$}

Una mención importante merece la migración oriunda de la región de Campania y dirigida hacia varios lugares del país andino. Nos capta la atención el hecho de que el primer campano registrado en 1899 sea un antepa-

72 Cappelli, 2005, 33.

73 Se registraron en 1925, cuando el hijo tenía 12 años de edad.

74 Véase nota 37.

75 Sobre este tema véase Sinardet, 1998; Arellano Paredes, 1998.

76 Véase nota 37.

77 Idem. 
sado (y homónimo) del hombre que ha alcanzado los honores de la crónica por sus fotografías del grupo de connacionales en el Ecuador de la década de los veinte. ${ }^{78}$ Conforme a lo que se reporta en la publicación del fotógrafo aficionado, la tienda de la familia —en la que él mismo trabajó- fue abierta en Guayaquil en 1880 y comerciaba con textiles de lino, seda, casimires y algodón. Posteriormente, el fotógrafo se mudó a vivir en la capital ecuatoriana y fue el mismo que abrió una filial de la tienda de Guayaquil en la ciudad de Quito en 1932. ${ }^{79}$ Otras fuentes reportan que la marca D'Aniello Hnos. \& Cía. fue fundada en 1911 y que para la década de los veinte era una de las más importantes casas de importación de Ecuador, siendo más del $80 \%$ del total de sus importaciones provenientes de Italia. ${ }^{80}$

En términos más generales, se destaca que entre los 68 campanos inscriptos en las representaciones diplomáticas, la mayoría, 26 individuos, provenían de Secondigliano (hoy englobada en la ciudad de Nápoles); de ellos, 16 estaban instalados en la ciudad de Quito y 7 en Guayaquil. ${ }^{81}$

Secundariamente, se destaca que aparecen únicamente cinco campanos (entre ellos los D'Aniello) que se han inscrito en el registro de Guayaquil en 1899, habiendo llegado todos entre 1878 y 1891. Las informaciones sobre las llegadas en periodos posteriores son más escasas, pero, analizando la fecha de emisión del pasaporte para emigrar, podemos ver un incremento de la migración entre la región de Campania, en particular Secondigliano, y la ciudad de Quito, a partir de los años veinte del siglo pasado. ${ }^{82}$

Se añade que, estudiando las fichas de los ítalo-ecuatorianos, solo cinco entre ellos son de origen campano, ${ }^{83}$ cuatro residen en Ambato, uno en Quito y uno en Guayaquil. Giuseppe di Donato es nacido en 1885 e inscrito en el registro de Quito en 1908; Luigi Antonio Rosania, nacido en 1885, inscrito en Quito en 1908; Pietro Raffone, nacido en 1907, residente en Ambato, inscrito en el registro de Quito; Gaetano Musella, nacido en 1883, residente en Ambato e inscrito en el registro de Quito; Giuseppe Renella, nacido en 1881, residente en Ambato e inscrito en el registro de Quito; Pietro Raffone, nacido en 1907, residente en Ambato e inscrito en el registro de Quito; Anna Miranda Stornaiolo, nacida en 1901, inscrita el 14 de junio

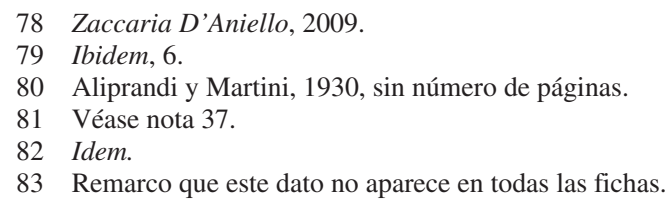

83 Remarco que este dato no aparece en todas las fichas. 
de 1932 y residente en Guayaquil; Maria Stornaiolo, nacida en 1927, probablemente la hija de la anterior Anna Miranda, ya que además se inscribieron en la misma fecha, aunque la segunda reside en Quito. ${ }^{84}$

Ahora bien, se trata de trayectorias con características bien diferentes de las de los ligures asentados en Guayaquil, presentando similitudes, por el contrario, con los piamonteses asentados en Ecuador, por lo que concierne a la distribución geográfica.

Igualmente, entre los oriundos de Piamonte hay pocos que figuren entre los pioneros; hay solo dos casos de inscritos en las representaciones diplomáticas en 1899, los otros lo hicieron todos en fechas posteriores. Un dato emerge con fuerza: entre los primeros que se inscribieron, Guayaquil aparece sin lugar a duda como destino privilegiado, con 25 individuos de 85 en total, mientras, con el trascurso del tiempo, a lo largo del siglo XX los destinos cambian y asume una importancia prominente la ciudad de Quito, destino para 41 individuos. ${ }^{85}$ Otra característica peculiar de este flujo es el abanico profesional. En este caso, a diferencia de los tres precedentes, no encontramos una clara especialización profesional o un claro predominio de los comerciantes, pudiendo rastrear albañiles, industriales, periodistas, mecánicos, estudiantes, docentes de arte religioso, religiosos, agricultores, orfebres, tejedores, etc. ${ }^{86}$

En el caso de la migración de Campania y de Piamonte, nos parece poder ver que, en el trascurso del tiempo, los flujos migratorios evolucionan y se diferencian las trayectorias geográficas, es decir que desde una concentración en Guayaquil en el siglo XIX se asiste a una diferenciación regional en cuanto al origen y a una ampliación del abanico de los destinos ecuatorianos en el siglo XX. Para entender este proceso, otra vez hay que hacer referencia a las características endógenas ecuatorianas. Fue finalmente en 1908 cuando se terminó la construcción de la línea de ferrocarril que unía Guayaquil y Quito, el Ferrocarril Transandino, conectando así las dos regiones y las dos economías diferentes y, por lo que hace a nuestro interés, facilitando el traslado y el posterior asentamiento de los inmigrantes, desde la costa hacia el interior del país andino.

Se finaliza este apartado con una observación sobre un flujo numéricamente poco significativo, pero interesante por sus características. Se ha

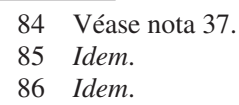


rastreado un pequeño grupo de mineros sardos que se dirigen hacia Zaruma, zona de explotación minera ecuatoriana, desvelando así la existencia de diferentes redes profesionales que superan las fronteras y los océanos. ${ }^{87}$

\section{Conclusiones}

Cerrando este trabajo, creo poder afirmar que la llegada de los italianos a Ecuador no se vincula con las políticas migratorias gubernamentales. Las tentativas de Ecuador para traer inmigrantes italianos no llegaron nunca más allá de la fase de planificación. Esto significa que nos encontramos frente a una inmigración «espontánea», es decir, no planificada por las autoridades ecuatorianas o italianas. En los casos que se han mostrado, es evidente la insuficiencia de explicaciones de las migraciones basándose en factores económicos (expulsión-atracción) o políticos (acuerdos entre países).

A través del estudio de las fichas se ha demostrado la importancia de las redes familiares y de paisanaje, que a menudo corresponden a redes profesionales, en la orientación de los inmigrantes hacia un lugar más que a otro. Se asiste, por ende, a una emigración desde precisos lugares de partida hacia un lugar de llegada y hacia un determinado empleo, reconfirmando, como en otros casos, la importante atracción que para los potenciales emigrantes representaban sus paisanos ya instalados en el exterior.

Además, en unos cuantos casos, se ha verificado que la inscripción en las representaciones diplomáticas italianas no se hacía inmediatamente al momento de la llegada a Ecuador, confirmando el escaso interés en mantener las conexiones formales con la madre patria, prefiriendo a ello las relaciones directas con los compatriotas emigrantes. La alternativa a las políticas oficiales, en cuanto a la envergadura de esta migración, parecen ser los lazos de amistad y familiares. En términos generales, hombres y mujeres fueron llegando a Ecuador a lo largo de la segunda mitad del siglo XIX de manera individual, acudiendo a la llamada de un pariente, un compaisano o un conocido.

A partir del análisis de los vínculos establecidos entre el lugar de origen y el de destino, se ha visto cómo las movilidades han conocido diferentes temporalidades. En la segunda mitad del siglo XIX hubo un momento

87 Idem. 
de aceleración cuando el flujo era hegemonizado por la presencia de la migración ligur, que se insertaba en el comercio de exportación del puerto de Guayaquil, durante la bonanza económica del auge del cacao. Luego se ha visto un decrecimiento en el siglo $\mathrm{XX}$, aunque nunca un bloqueo, y aunque los ligures (comerciantes) no desaparecen del flujo, el abanico regional y profesional empieza a ampliarse. A la vía hacia Ecuador abierta por los ligures empiezan a sumarse otras poblaciones, como las lucano-calabresas, de larga tradición migratoria hacia otros destinos y de precisa especialización profesional. Finalmente, hemos visto cómo con la abertura del ferrocarril los flujos ya no se circunscriben a la costa, empezando a dirigirse hacia la capital, Quito.

Es importante subrayar que el mundo urbano ecuatoriano representó una fuerte atracción para los italianos, independientemente de la zona de partida, fuese rural o urbana. Sin lugar a duda, destaca que son rarísimos los casos de italianos que se dedicaron a trabajos agrícolas, privilegiando a menudo las actividades económicas a desarrollar en el principal puerto ecuatoriano, y más en general en el comercio al por menor, al por mayor y de exportación.

Igualmente, se ha observado que las condiciones económico-sociales en el contexto de llegada condicionaron la trayectoria de estos inmigrantes. El auge del cacao favoreció la entrada en el sector del comercio a aquellos primeros «pioneros» ligures del siglo XIX, que pudieron así ver aumentar su capital económico y social. Los que llegaron después no tuvieron la misma suerte, teniendo que enfrentarse a una fase económica negativa y a un mercado para la exportación saturado, y de ahí la progresiva diferenciación profesional de quienes llegaron en el siglo XX.

Recibido el 16 de octubre de 2018

Aceptado el 18 de enero de 2019

\section{Referencias bibliográficas}

Aliprandi, Ermenegildo y Martini, Virgilio, Gli italiani in Equatore. Rassegna delle vite e delle opera della stirpe italica in terra ecuatoriana, Guayaquil, Artes Gráficas «Senefelder», 1930.

Aliprandi, Ermenegildo y Martini, Virgilio, Anuario Ecuatoriano, Guayaquil, Aliprandi y Martini editores, 1935. 
Anderson, Benedict, Imagined Communities: Reflections on the Origin and Spread of Nationalism, London, Verso, 1991.

Annoni, Antonio Marcello, «L'Italia e l'Ecuador», Bollettino della Reale Società Geografica Italiana, LIV, LVII, 1920, 244-248.

Arellano Paredes, Víctor Hugo, «La aristocracia ecuatoriana en el París de inicio del siglo XX», Revista del Instituto de Historia Marítima, 23, Guayaquil, 1998, 159-188.

Ayala Mora, Enrique, Ecuador en el Siglo XIX - Estado Nacional, Ejército, Iglesia y Municipio, Quito, Universidad Andina Simón Bolívar, Corporación Editora Nacional, 2011.

Bianchi Tonizzi, Maria Elisabetta, «Il movimento dell'emigrazione nel porto di Genova dalla metà dell'Ottocento agli anni trenta del nostro secolo», en Maiello, Adele (ed.), L'emigrazione nelle Americhe dalla provincia di Genova, vol. 4, Questioni di storia sociale, Bologna, Patron editore, 1992, 21-43.

Bonfiglio, Giovanni, Gli italiani nella società peruviana. Una visione storica, Torino, Edizioni della Fondazione Giovanni Agnelli, 1999.

Braudel, Fernand, Civiltà e Imperi del Mediterraneo nell'età di Filippo II, Torino, Einaudi, 2010.

Bustamante, Jesús; Giraudo, Laura y Mayer, Leticia, La novedad estadística. Cuantificar, cualificar y transformar las poblaciones en Europa y América Latina, siglos XIX y XX, Madrid, Ediciones Polifemo, 2014.

Cagiao Vila, Pilar, Cinco siglos de emigración gallega a América, Madrid, Quinto Centenario, 1992.

Cappelli, Vittorio, «Verso le Americhe. Alle origini dell'emigrazione transoceanica in Calabria e in Lucania», Apollinea, 6, 2005, 32-37.

Capua, Alberto di, «Brevi cenni sull'emigrazione di ebrei italiani in Ecuador (1938-1945)», en Smolensky, Eleonora M. y Vigevani Jarach, Vera, Tante voci, una storia. Italiani ebrei in Argentina 1938-1948, Bologna, Il Mulino, 1998, 433-450.

Carletti, Tommaso, «Gli italiani all'Equatore», Bollettino dell'emigrazione, 5, 1907, 46-50.

Colucci, Michele y Sanfilippo, Matteo, Guida allo studio dell'emigrazione italiana, Viterbo, Sette Città, 2010.

Corti, Paola y Sanfilippo, Matteo, L'Italia e le migrazioni, Milano, Laterza, 2012.

Crawford de Roberts, Louis, El Ecuador en la época cacaotera. Respuestas locales al auge y colapso en el ciclo mono exportador, Quito, Editorial Universitaria, 1980.

Devoto, Fernando, Storia degli italiani in Argentina, Roma, Donzelli Editore, 2007.

Dizionario storico biografico dei Liguri in America latina da Colombo a tutto il Novecento, Ancona, Fondazione Casa America, Affinità Elettive, 2006, vol. II. 
Estrada, Jenny, Los Italianos de Guayaquil, Guayaquil, Sociedad Italiana «Garibaldi», 1993.

Estrada Turra, Baldomero (ed.), Inmigración española en Chile, Santiago, Universidad de Santiago de Chile / Universidad de Valparaíso / Universidad Metropolitana de Ciencias de la Educación / Embajada de España, 1994.

González Martínez, Elda E., La inmigración esperada: la política migratoria brasileña desde João VI hasta Getulio Vargas, Madrid, Consejo Superior de Investigaciones Científicas, 2003.

Guarnieri Calò Carducci, Luigi, Dizionario storico-biografico degli Italiani in Ecuador e in Bolivia, Bologna, Il Mulino, 2001.

Guerra, François-Xavier, México: del antiguo Régimen a la Revolución, México, Fondo de Cultura Económica, 1988.

Jurado Noboa, Fernando, «Los italianos en Guayaquil entre 1628 y 1902», Historia Marítima, 17, Guayaquil, 2002, 55-68.

León, Francisco Javier, Exposición del Ministro del Interior y Relaciones Exteriores dirigida al Congreso Constitucional de 1871, Quito, Imprenta Nacional, 1871.

Levi, Giovanni, «Famiglie contadine nella Liguria del Settecento», Miscellanea Storica Ligure, V, 2, 1973, 207-290.

Maiello, Adele, «L'emigrazione dal Chiavarese: sue origini e caratteristiche», en Scritti in onore del Prof. Paolo Emilio Taviani. II. Diritto, Storia e Scienza Politiche. Annali della Facoltà di Scienza Politiche dell'Università di Genova, XII, 1986, 159-183.

Maitron, Jean y Pennetier, Claude (dirs.), Dictionnaire Biographique du Mouvement Ouvrier Français (DBMOF), Paris, Editions Ouvrières / Editions de l'Atelier, 1964-1997, 44 vols.

Martínez Riaza, Ascensión, «A pesar del gobierno». Españoles en el Perú, 18791939, Madrid, Consejo Superior de Investigaciones Científicas, 2006.

Martínez Rodríguez, Marcela, Colonizzazione al Messico! Las colonias agrícolas de italianos en México, 1881-1910, México, El Colegio de San Luis / El Colegio de Michoacán, 2013.

Mazeres, Javier, «En la mitad de los mundos: la presencia migratoria española en Quito y Ecuador», en Ramírez, Jacques (ed.), Ciudad-Estado, inmigrantes y política: Ecuador, 1890-1950, Quito, IAEN, 2012, 53-96.

Mera, J. T., Informe Consular. Población e Inmigración de Italia. Comercio, Navegación e Industria. Relaciones Comerciales Ítalo-ecuatorianas, Génova, Stabilimento Tipografico Unione Genovese, 1907.

Mezzadra, Sandro, Diritto di fuga. Migrazioni, cittadinanza, globalizzazione, Verona, Ombrecorte, 2001.

Molinari, Augusta, «Storia e storie di emigrazione dal Ponente ligure. Alcuni percorsi di ricerca», Recherches régionales, 132, 1995, en https://www.departe 
ment06.fr/documents/Import/decouvrir-les-am/rr132-1995-07.pdf [Consultado: 30/09/2018].

Ostuni, Maria Rosaria, «Leggi e politiche di governo nell'Italia liberale e fascista», en Bevilacqua, P.; De Clementi, A. y Franzina, E. (eds.), Storia dell'emigrazione italiana. Partenze, Roma, Donzelli Editore, 2001, 309-321.

Pagnotta, Chiara, Situando los márgenes de la Nación. Los italianos en Ecuador (siglo XIX-XX), Quito, Abya-Yala / TEIAA, 2016.

Pagnotta, Chiara, «El exilio errante de los republicanos españoles entre República Dominicana y Ecuador (1940-1942)», en Beneduzi, Luis Fernando (ed.), Autoritarismos en el mundo Ibérico e Iberoamericano, Toulouse, Méridiennes / Les Presses Universitaires du Midi, en prensa [2019].

Pagnotta, Chiara, «Ecuador, los inmigrantes y la propaganda fascista (1922-1938)», Revista de Indias, en prensa [2020].

Ponce, Nicolás Clemente, Informe del Ministerio de Relaciones Exteriores. Junio de 1920 - junio de 1921, Quito, Imprenta y Encuadernación Nacionales, 1921.

Porcella, Marco, «Da birbanti a emigranti. Itinerari della povertà contadina», en La via delle Americhe. L'emigrazione ligure tra evento e racconto. Catalogo della mostra, Genova, SAGEP editrice, 1989, 37-42.

Prieto, Mercedes, «El Estado ecuatoriano a mediados del siglo XX: el censo de la población y la familia indígena», Revista Europea de Estudios Latinoamericanos y del Caribe, 99, 2015, 29-46.

Quintero, Rafael y Silva, Erika, Ecuador: una nación en ciernes, Quito, Editorial Universitaria, 2001, vol. I.

Sanfilippo, Matteo, Problemi di storiografia dell'emigrazione italiana, Viterbo, Sette Città, 2002.

Schröter, Bernd y Büschges, Christian (eds.), Beneméritos, aristócratas y empresarios. Identidades y estructuras sociales de las capas altas urbanas en América Latina, Madrid / Frankfurt am Main, Iberoamericana / Vervuert, 1999.

Sinardet, Emmanuelle, «El mito de París y la oligarquía cacaotera en el Ecuador (1895-1925)», Revista del Instituto de Historia Marítima, 23, Guayaquil, 1998, 147-158.

Sori, Ercole, L'emigrazione italiana dell'Unità alla seconda guerra mondiale, Bologna, Il Mulino, 1979.

Stone, Laurence, «Prosopography», Daedalus, 100, 1, 1971, 46-79.

Vangelista, Chiara, Le braccia per la fazenda. Immigrati e caipiras nella formazione del mercato del lavoro paulista (1850-1930), Milano, Franco Angeli, 1982.

Zaccaria D'Aniello: La inmigración italiana en Ecuador en los años 20, Quito, Embajada de Italia, 2009. 